\title{
Selenium supplementation and autoantibody titers in Graves' disease
}

Marc Gregory Yu MD, Antonio Faltado Jr. MD, Laura Rosario Acampado MD

Section of Endocrinology, Diabetes, and Metabolism, Department of Medicine University of the Philippines- Philippine General Hospital Manila, Philippines

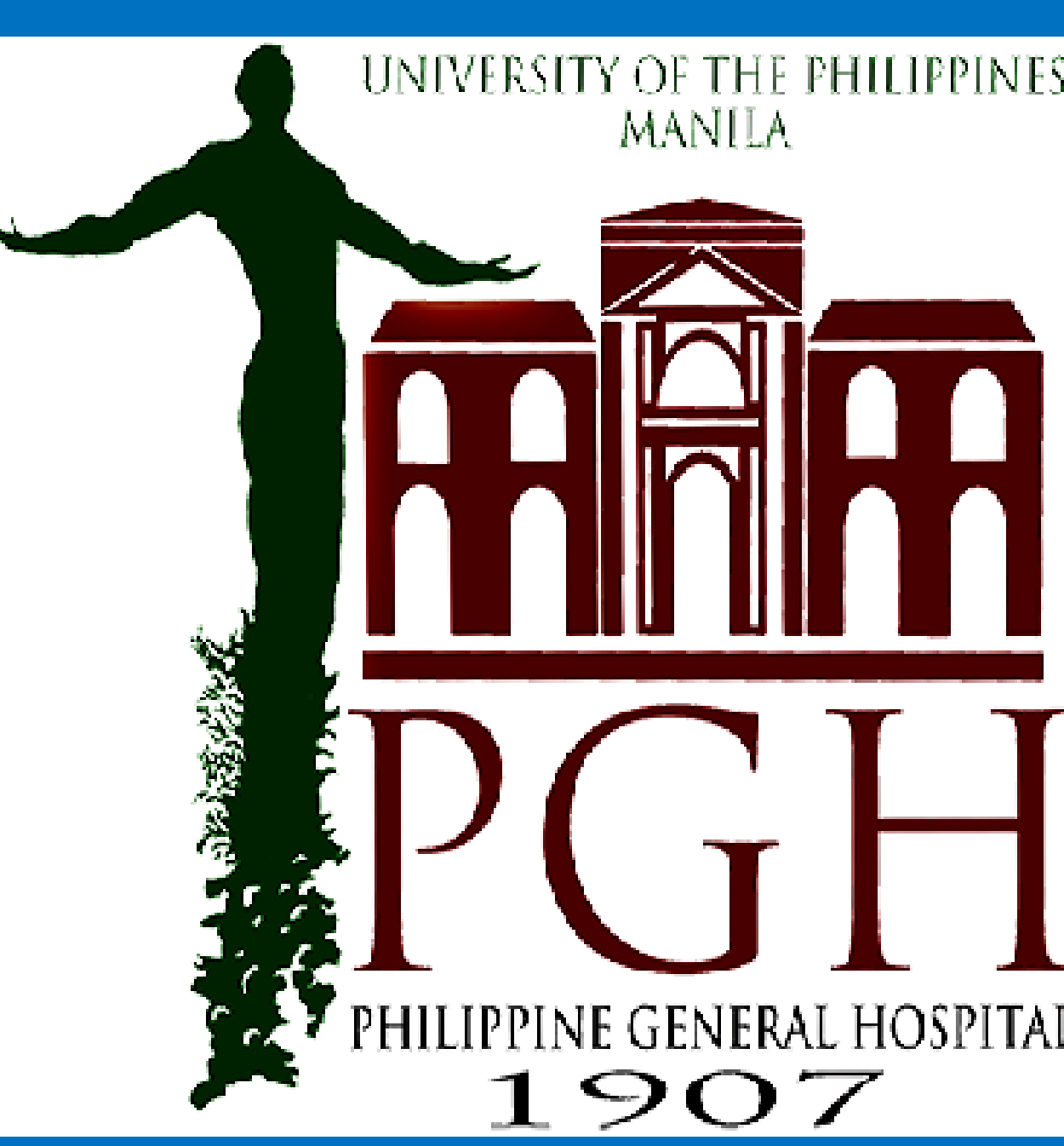

\section{Abstract}

Objectives: To evaluate the efficacy of Se supplementation in patients with GD and GO in terms of changes in ocular and systemic signs and symptoms, health-related quality of life (HRQoL), and selenoprotein, thyrotropin, thyroid hormone, and autoantibody levels.

Methods: RCTs evaluating the efficacy of Se supplementation in adult patients with GD and active GO, versus placebo or an alternative drug and on top of standard therapy, were included. A literature search was performed by two independent authors with eligible studies undergoing a validity screen. Data extraction of selected studies was done using a data extraction form, with statistical analysis using RevMan 5.1 software. Results were presented as mean differences, standard errors, and 95\% confidence intervals, and graphically presented as forest plots. Estimates were calculated using the inverse variance method for continuous variables and pooled using the fixed effects model. $\mathrm{I}^{2}$ and $\mathrm{Chi}^{2}$ tests were used to assess heterogeneity.

Results: Fourteen studies were initially retrieved for consideration, but only two trials were ultimately included. Both had good methodologica quality and totaled 197 patients with GD and nonsevere GO. The only available common outcomes of interest were changes in TRAB and TPOAB titers. No statistically significant difference was found in TRAB $(95 \% \mathrm{Cl},-1.38[-3.19,0.44]$, $\mathrm{p}=0.14)$ as well as in TPOAB $(95 \% \mathrm{Cl}, 36.66$ [$32.56,105.88], p=0.3$ ) titers on follow up among those given Se supplementation as compared to placebo. No significant heterogeneity was found in either the TRAB $\left(I^{2}=36 \%\right)$ or TPOAB $\left(I^{2}=0 \%\right)$ analysis.

Conclusions: This is the first meta-analysis summarizing the current available data on the efficacy of Se supplementation in patients with GD and active non-severe GO. Se supplementation in these patients was not associated with statistically significant differences in both TRAB and TPOAB titers on follow up. Larger studies are recommended to strengthen these findings.

\section{Background}

Selenium (Se), a trace mineral with antioxidative properties, has been proposed by studies to be potentially beneficial in patients with Graves' disease (GD), especially those with active Graves' ophthalmopathy (GO).

\section{Objectives}

General: To evaluate efficacy of Se supplementation in patients with GD and GO Specific: To evaluate changes in:

Ocular and systemic signs and symptoms

2. Health-related quality of life

3. Selenoprotein, thyrotropin, thyroid hormone, and autoantibody levels

\section{Methods}

\section{Databases}

- MEDLINE, Embase, ClinicalTrials.gov,

Google Scholar, Cochrane Central Register of Controlled Trials

References of articles and individual authors

\section{Keywords}

"selenium", "selenite", "selenoprotein"

"thyroid", "orbitopathy", "ophthalmopathy"

"hyperthyroidism", "Graves' disease", "thyrotoxicosis"

thyroid-related eye disease", "Basedow disease"

\section{Inclusion Criteria}

- RCTs evaluating efficacy of Se supplementation on top of standard therapy in adult GD patients with active GO

- Versus either placebo or alternative controller

- Outcomes: clinical activity of GO as measured via objective examination or symptom scores; levels of selenium or selenoproteins, $\mathrm{TSH}$, thyroid hormones TRAB and TPOAB, HRQoL

No restrictions on language, ethnicity or gender

\section{Exclusion Criteria}

- Pregnancy

- Comorbid systemic or ocular disease

Severe GO requiring steroid use at outset

Previous or ongoing use of Se supplements

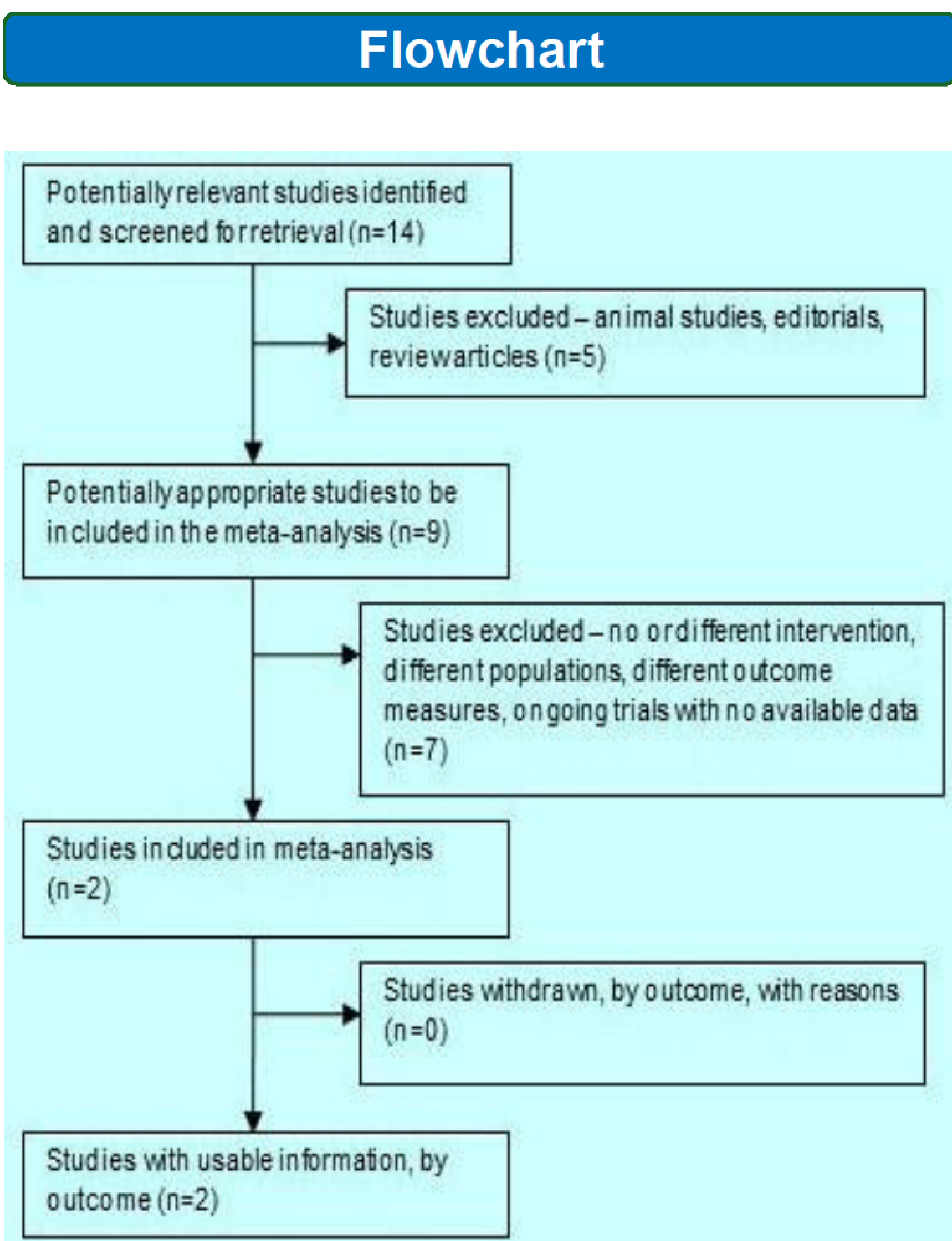

Figure 1. Flowchart of methodology used to arrive at the studies included in the analysis.

\section{Results}

\begin{tabular}{|c|c|c|}
\hline Study & $\begin{array}{c}\text { Marcocci et al (EuGOGO Trial) } \\
2011\end{array}$ & $\begin{array}{c}\text { Calissendorff et al } \\
2015\end{array}$ \\
\hline Randomization & Adequate & Adequate \\
\hline $\begin{array}{c}\text { Allocation } \\
\text { Concealment }\end{array}$ & Yes & Yes \\
\hline $\begin{array}{c}\text { Baseline } \\
\text { Characteristics }\end{array}$ & No significant difference & No significant difference \\
\hline Blinding & Double blind & Double blind \\
\hline Follow-up Rates & Adequate & Adequate \\
\hline
\end{tabular}

Table 1. Methodological assessment of the quality of studies included in the review.

\begin{tabular}{|c|c|c|}
\hline Study & $\begin{array}{l}\text { Marcocci et al (EuGOGO Trial) } \\
2011\end{array}$ & $\begin{array}{l}\text { Calissendorff et al } \\
2015\end{array}$ \\
\hline Title & $\begin{array}{l}\text { Selenium and the course of mild } \\
\text { Graves' ophthalmopathy }\end{array}$ & $\begin{array}{l}\text { A prospective investigation of Graves' } \\
\text { disease and selenium: thyroid hormones, } \\
\text { auto-antibodies, and self-rated symptoms }\end{array}$ \\
\hline Design & RCT & RCT \\
\hline Therapy Duration & 6 months & 9 months \\
\hline Sample Size & 159 & 38 \\
\hline Population & $\begin{array}{l}\text { Adult GD patients aged } 18-70 \\
\text { with mild } G 0<18 \text { mo duration }\end{array}$ & $\begin{array}{l}\text { Adult GD patients aged 18-55 without } \\
\text { severe GO }\end{array}$ \\
\hline Outcomes & $\begin{array}{l}\text { Eye evaluation, GO-QoL score, } \\
\text { clinical activity score, diplopia } \\
\text { score, TRAB, TPOAB }\end{array}$ & $\begin{array}{c}\text { Selenoprotein concentration, self-rated } \\
\text { symptom score, anxiety and depression } \\
\text { score, TSH, ft4, fT3, TRAB, TPOAB }\end{array}$ \\
\hline Intervention & Se 200 ug/day & Se 200 ug/day \\
\hline Comparator & Placebo, Pentoxifylline & Placebo \\
\hline
\end{tabular}

Table 2. Characteristics of the studies included in the review.

\begin{tabular}{|c|c|}
\hline Study & Reason for Exclusion \\
\hline Watt 2013 & Ongoing trial, no data yet available \\
\hline Wertenbruch 2007 & Case control design, no intervention \\
\hline Khong 2014 & Case control design, no intervention \\
\hline Pedersen 2013 & Cross-sectional design, no intervention \\
\hline Vrca 2003 & Different intervention and outcome measures \\
\hline Smith 2011 & Review article \\
\hline Duntas 2011 & Review article \\
\hline Dharmasena 2014 & Review article \\
\hline Sturniolo 2013 & Editorial \\
\hline Toulis 2010 & Different disease population \\
\hline Fan 2014 & Different disease population \\
\hline Xu 2011 & Animal study \\
\hline
\end{tabular}

Table 3. List of excluded studies and reasons for exclusion.

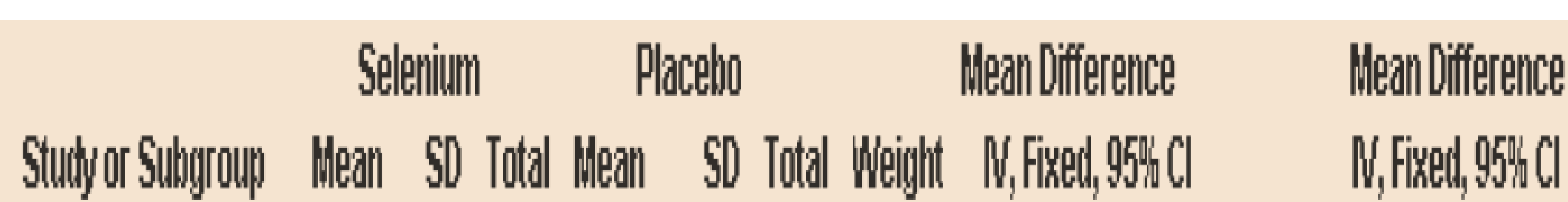

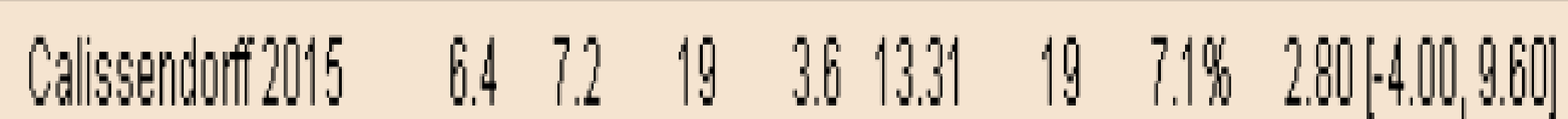

$\begin{array}{llllllll}\text { Marcocici } 2011 \quad 8.7576 & 54 & 10.4 & 3.93 & 50 & 92.9 \% & -1.70[-3.56,0.18]\end{array}$

\section{Total $\mid 050)(1)$ \\ $73 \quad 60100,046-1,38[-3,19,0,44]$}

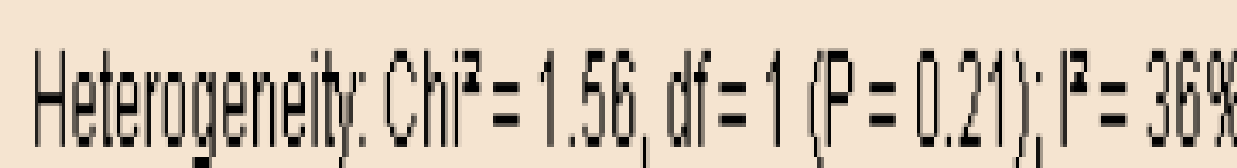

Tess tor overall entect $z=1.49(P=0.14)$

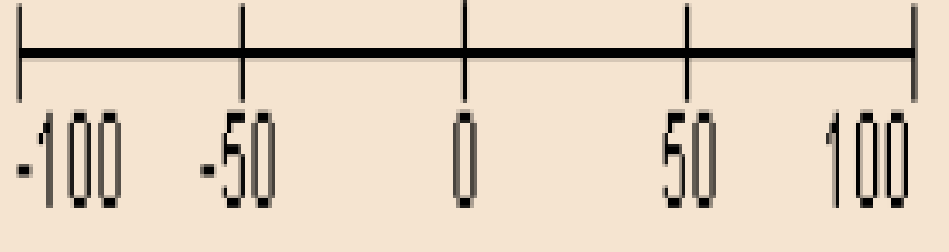

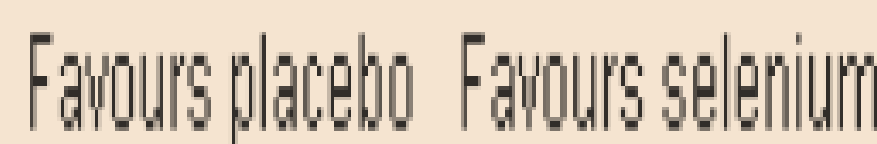

Figure 2. Mean difference in TRAB titers between the Se and placebo groups.

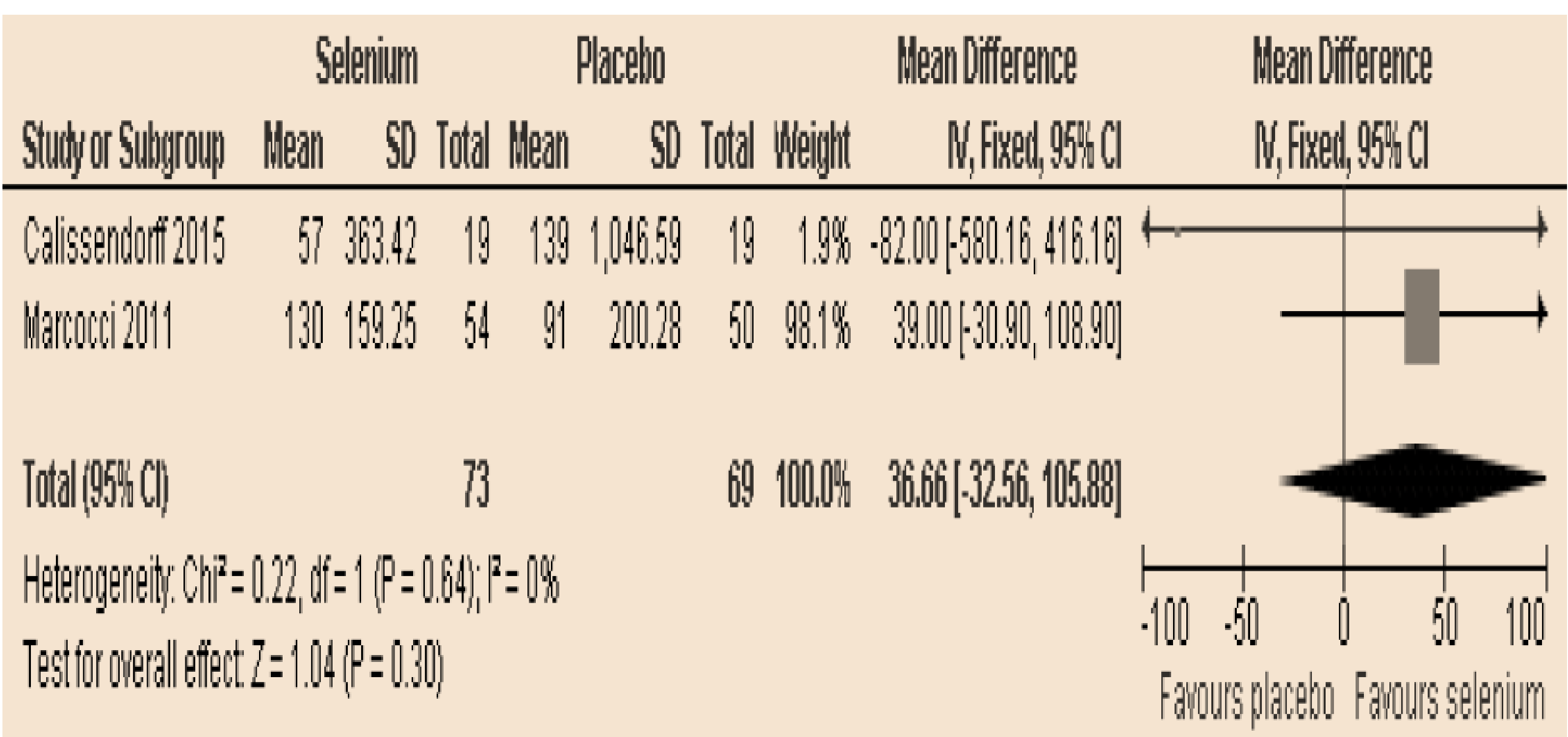

Figure 3. Mean difference in TPOAB titers between the Se and placebo groups.

\section{Conclusions}

Se supplementation in patients with $G D$ and non-severe $G O$ was not associated with significant differences in TRAB and TPOAB titers.

More studies with larger populations and more clinical outcomes are recommended. 